\title{
The domestication of Cucurbita argyrosperma as revealed by the genome of its wild relative
}

\author{
Josué Barrera-Redondo (1)', Guillermo Sánchez-de la Vega (1)', Jonás A. Aguirre-Liguori (i)',

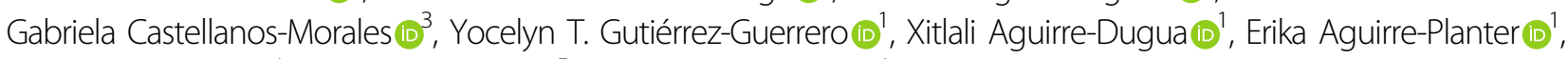 \\ Maud I. Tenaillon (10), Rafael Lira-Saade $\mathbb{1 0}^{5}$ and Luis E. Eguiarte (1)
}

\begin{abstract}
Despite their economic importance and well-characterized domestication syndrome, the genomic impact of domestication and the identification of variants underlying the domestication traits in Cucurbita species (pumpkins and squashes) is currently lacking. Cucurbita argyrosperma, also known as cushaw pumpkin or silver-seed gourd, is a Mexican crop consumed primarily for its seeds rather than fruit flesh. This makes it a good model to study Cucurbita domestication, as seeds were an essential component of early Mesoamerican diet and likely the first targets of humanguided selection in pumpkins and squashes. We obtained population-level data using tunable Genotype by Sequencing libraries for 192 individuals of the wild and domesticated subspecies of C. argyrosperma across Mexico. We also assembled the first high-quality wild Cucurbita genome. Comparative genomic analyses revealed several structural variants and presence/absence of genes related to domestication. Our results indicate a monophyletic origin of this domesticated crop in the lowlands of Jalisco. We found evidence of gene flow between the domesticated and wild subspecies, which likely alleviated the effects of the domestication bottleneck. We uncovered candidate domestication genes that are involved in the regulation of growth hormones, plant defense mechanisms, seed development, and germination. The presence of shared selected alleles with the closely related species Cucurbita moschata suggests domestication-related introgression between both taxa.
\end{abstract}

\section{Introduction}

Domestication is an evolutionary process where human societies select, modify and eventually assume control over the reproduction of useful organisms. A mutualistic relationship emerges from this interaction, where humans exploit a particular resource of interest, while the domesticated organism benefits from increased fitness and extended geographical range ${ }^{1,2}$. This is well illustrated

\footnotetext{
Correspondence: Josué. Barrera-Redondo (josue_barrera@comunidad.unam.mx) or Rafael Lira-Saade (rlira@unam.mx) or Luis E. Eguiarte (fruns@unam.mx) ${ }^{1}$ Departamento de Ecología Evolutiva, Instituto de Ecología, Universidad Nacional Autónoma de México, Circuito Exterior s/n Anexo al Jardín Botánico, 04510 Ciudad de México, México

'Department of Ecology and Evolutionary Biology, University of California, Irvine, CA 92697, USA

Full list of author information is available at the end of the article These authors contributed equally: Josué Barrera-Redondo, Guillermo

Sánchez-de la Vega
}

in Cucurbita L. (pumpkins, squashes, and some gourds), where human-guided domestication and breeding have considerably extended their distribution despite the extinction of their natural dispersers (e.g., mastodons and similar megafauna) ${ }^{3}$. Today, Cucurbita stand as successful crops grown and consumed worldwide, with a global annual production of $\sim 24$ million tons ${ }^{4}$.

With ca. 21 taxa, the Cucurbita genus has experienced independent domestication events in five species ${ }^{5,6}$. Each Cucurbita crop experienced a unique selection for specific traits, predominantly defined by the nutritional and cultural needs of early human populations in America ${ }^{7}$. However, many domestication traits are common to domesticated Cucurbita, including the loss of bitter compounds (cucurbitacins), the loss of physical defense mechanisms (e.g., urticating trichomes), the loss of seed

\section{(c) The Author(s) 2021}

(c) (i) Open Access This article is licensed under a Creative Commons Attribution 4.0 International License, which permits use, sharing, adaptation, distribution and reproduction in any medium or format, as long as you give appropriate credit to the original author(s) and the source, provide a link to the Creative Commons license, and indicate if changes were made. The images or other third party material in this article are included in the article's Creative Commons license, unless indicated otherwise in a credit line to the material. If material is not included in the article's Creative Commons license and your intended use is not permitted by statutory regulation or exceeds the permitted use, you will need to obtain permission directly from the copyright holder. To view a copy of this license, visit http://creativecommons.org/licenses/by/4.0/. 
dormancy, the enlargement of fruits and seeds, and the diversification of fruit morphology ${ }^{4,8}$.

The initial steps of Cucurbita domestication were most likely directed towards seed rather than flesh consumption'. Seeds are rich in both carbohydrates and fatty acids, and cucurbitacins can be removed through boiling and washing; processes that are still employed for the consumption of wild Cucurbita seeds in Western Mexico ${ }^{7}$. Because the cultivation of $C$. argyrosperma (pipiana squash, cushaw pumpkin, or silver-seed gourd) is directed towards seed production rather than fruit flesh, it stands as an excellent model to investigate the early steps of Cucurbita domestication. Cucurbita argyrosperma subsp. argyrosperma (argyrosperma hereafter) was domesticated in Mesoamerica from its wild relative Cucurbita argyrosperma subsp. sororia (sororia hereafter), according to archaeological and genetic evidence ${ }^{6,10,11}$. Argyrosperma exhibits morphological differences from sororia, including larger fruits, larger seeds, and lack of urticating trichomes (Fig. 1). The earliest archaeological record of argyrosperma is presumed to be from 8700 -year-old phytoliths in the Central Balsas Valley (Guerrero), although its taxonomic identity remains uncertain ${ }^{10}$. C. argyrosperma is a monoecious outcrossing species and gene flow has been previously described between the domesticated and wild subspecies ${ }^{12}$. Both subspecies are sympatric throughout the Pacific Coast of Mexico and Central America, with a few populations scattered in the coast of

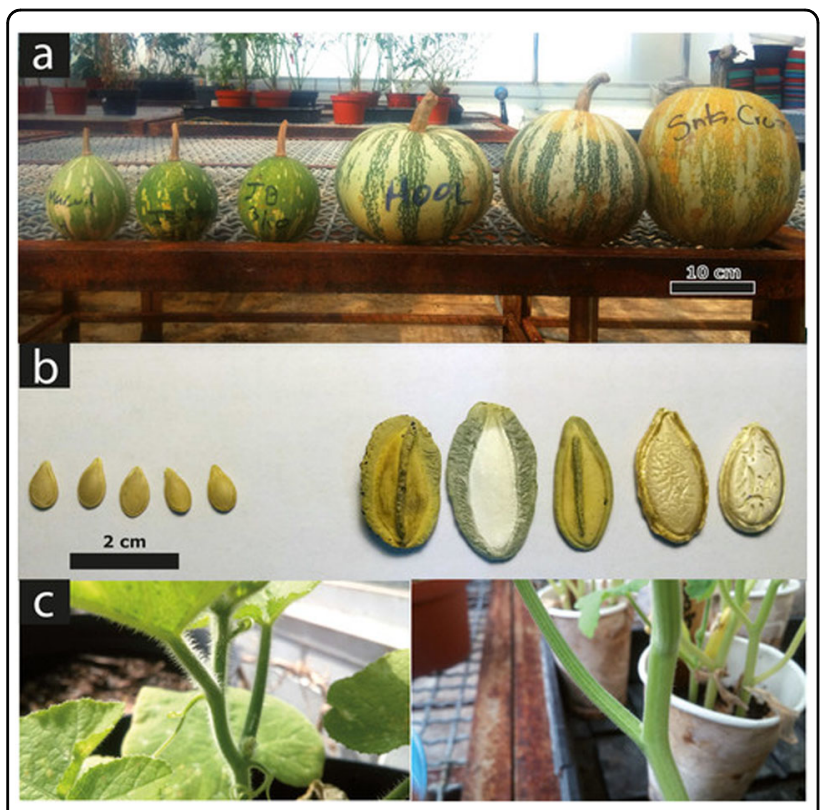

Fig. 1 Some morphological differences between C. argyrosperma subsp. sororia (left) and C. argyrosperma subsp. argyrosperma (right). Differences in a fruit size, $\mathbf{b}$ seed size and shape, $\mathbf{c}$ and in the presence of urticating trichomes the Gulf of Mexico ${ }^{11,13}$. The domesticated taxon is also distributed in the Yucatan Peninsula, where its wild counterpart is absent ${ }^{11}$.

Despite the economic importance and the growing genomic resources in Cucurbita species, ${ }^{8,13}$, studies aimed at understanding their domestication are still lacking. To start filling this gap, we report here the first genome assembly of the wild relative sororia, which complements the existing assembly of argyrosperma ${ }^{14}$. The comparison between the genomes allowed us to find genomic structural variants between both subspecies. We characterized a large sample of argyrosperma landraces (117 individuals from 19 locations) and sororia accessions (50 individuals from 4 locations) using genome-wide data to investigate their demographic history and propose a domestication scenario. We also performed selection scans throughout the genome of C. argyrosperma to detect candidate regions associated with the domestication of this species.

\section{Results}

Genome assembly of C. argyrosperma subsp. sororia

We sequenced the genome of a wild individual of subspecies sororia using Illumina HiSeq4000 (213x coverage) and PacBio Sequel (75.4x coverage). The genome was assembled in 828 contigs with an N50 contig size of $1.3 \mathrm{Mbp}$ and an L50 of 58 contigs (Table S1). A BUSCO analysis ${ }^{15}$ against the embryophyte odb9 database detected $92.8 \%$ of complete BUSCOs, $1.2 \%$ fragmented BUSCOs, and $6.0 \%$ missing BUSCOs within the genome assembly, similarly to other Cucurbita genome assemblies $^{14,16}$. We predicted 30,592 protein-coding genes within the genome assembly using BRAKER2 ${ }^{17}$. The BUSCO completeness of the gene predictions $(92.5 \%$ complete BUSCOs, $3.1 \%$ fragmented BUSCOs) is comparable to that of the genome assembly and that of other genome annotations ${ }^{14,16}$, despite using RNA-seq data of a different individual from which the genome was assembled (see "Methods"). Around $35.8 \%$ of our sororia genome assembly is composed of transposable elements (TEs), slightly higher than the $34.1 \%$ of TEs found in a previous argyrosperma assembly ${ }^{14}$.

The genome of argyrosperma was previously assembled in 920 scaffolds $^{14}$, so we aimed at improving the assemblies for both the sororia and the argyrosperma genomes using a reference-guided scaffolding step against the genome assembly of C. moschata ${ }^{16}$. We anchored $99.97 \%$ of the argyrosperma genome assembly and $98.8 \%$ of the sororia genome assembly into 20 pseudomolecules using $\mathrm{RaGOO}^{18}$, which corresponds to the haploid chromosome number in Cucurbita $^{19}$. Both assemblies show high synteny conservation across the genus (Fig. S1) and confirm a previously reported inversion in chromosome four that is shared with C. moschata ${ }^{16}$. 

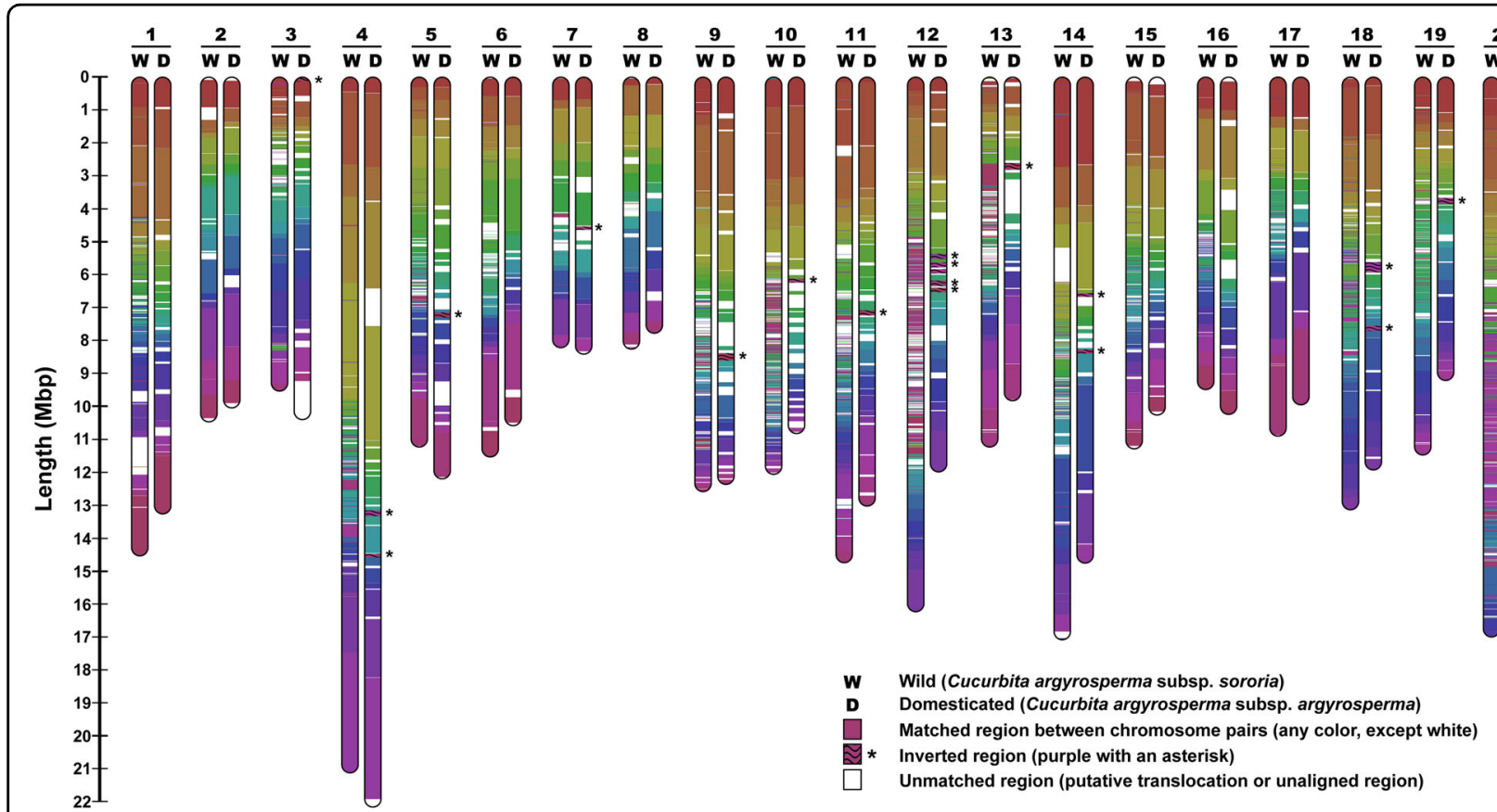

Fig. 2 Chromosome map representing the matching regions and putative structural variants between the genome assemblies of C. argyrosperma subsp. sororia (wild) and C. argyrosperma subsp. argyrosperma (domesticated). Matching colors represent the aligned homologous regions between both genomes, while white segments represent regions that could not be aligned to the other genome. Inverted regions are highlighted with an asterisk

\section{Structural variants between the wild and domesticated genomes}

We compared the genome of argyrosperma against the genome of sororia (Fig. 2). Some of the centromeres in the genome of sororia were larger than in argyrosperma, possibly due to a better assembly of the repetitive regions. We found 443 high-confidence structural variants (SVs) such as copy-number variants (CNVs), inversions, and translocations between the wild and the domesticated genomes (Table S2). We also found several regions that could not be aligned between both genomes (Table S2). The size of the sororia genome assembly is $\sim 254 \mathrm{Mbp}$, 9.23\% larger than the genome assembly of argyrosperma ${ }^{14}$, which could be partially explained by these SVs. We found that two copies of thaumatin-like protein 1a (TL1) and auxin-responsive protein SAUR32 were disrupted by inversions in the argyrosperma genome. We also found a CNV loss spanning the transcription factor PIF1 and a translocation containing the gene LONG AFTER FAR-RED 3 (LAF3). The genomes of argyrosperma and sororia share some common genes within their unaligned regions, such as microtubule-associated proteins and genes related to tryptophan biosynthesis (Table S2), suggesting that those regions contain highly divergent sequences and are not limited to presence/ absence variants. However, other unaligned regions contain more genes in sororia than in argyrosperma, including the major pollen allergen Ole e 6 (OLE6), some proteolytic enzymes and sucrose biosynthetic genes that are absent in the argyrosperma genome (Table S2), suggesting that presence/absence variants are also included within the unaligned regions.

\section{Population data and SNP genotyping}

We used samples previously collected throughout Mexico $^{11}$ corresponding to 117 individuals of argyrosperma, 50 individuals of sororia, 19 feral individuals of argyrosperma previously reported to have a semi-wild phenotype and a cultivated genotype based on microsatellite data ${ }^{11}$ and 6 individuals of $C$. moschata (the domesticated sister species of $C$. argyrosperma), that were used as outgroups (Table S3). The samples were sequenced using the tunable Genotype by Sequencing (tGBS) method ${ }^{20}$ to obtain genome-wide genetic information of the $C$. argyrosperma populations. The reads were quality-filtered and mapped against the genome assemblies of argyrosperma and sororia to predict single nucleotide polymorphisms (SNPs) and assess possible reference biases in the SNP prediction. Using the reference genome of argyrosperma, we obtained an initial dataset consisting of 12,813 biallelic SNPs with a mean read depth of 50 reads per SNP and a minor allele frequency (MAF) of at least $1 \%$ (13k dataset). We also mapped the whole-genome Illumina reads of 


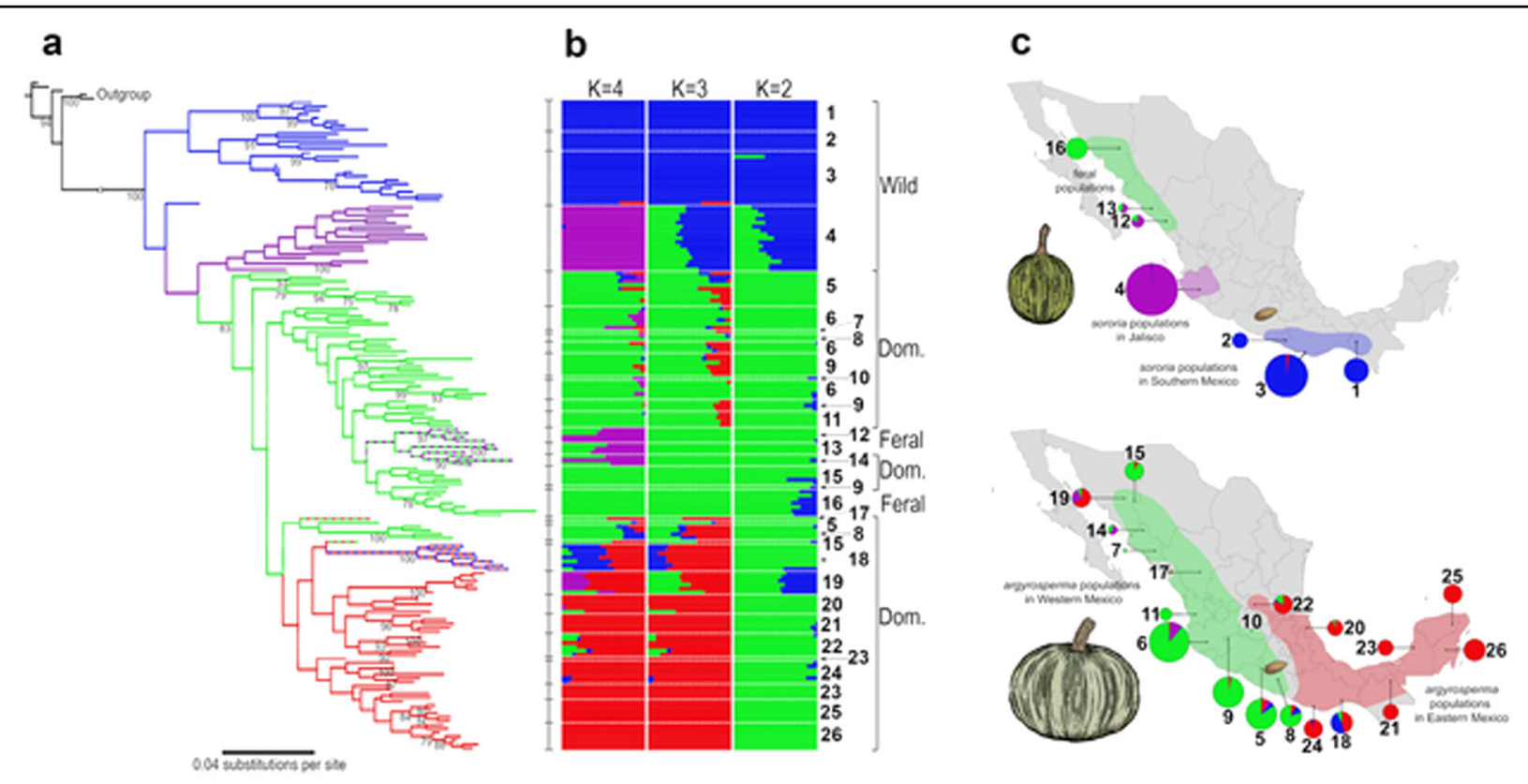

Fig. 3 Genetic structure and phylogenetic relationships between the wild and domesticated populations of Cucurbita argyrosperma based on 2861 SNPs. a Maximum-Likelihood tree with 100 bootstraps (only bootstrap values > 70 shown). b ADMIXTURE analysis using $K$ values ranging from 2 to 4. c Geographic distribution of the wild (top) and domesticated (bottom) populations, with pie chart colors representing the ADMIXTURE assignation of the individuals in $K=4$ genetic groups (size of pie charts proportional to sample size). The seed in the maps represents the earliest archaeological record of argyrosperma from Xihuatoxtla, Guerrero (dated 8700 years BP) ${ }^{10}$

argyrosperma and sororia, as well as the whole-genome sequencing of a $C$. moschata individual and a $C$. okeechobeensis subsp. martinezii individual (a closely related wild Cucurbita species), against the reference genome of argyrosperma to obtain a dataset of 11,498,421 oriented biallelic variants (SNPs and indels) across the genome that was used to assess introgression and incomplete lineage sorting.

\section{Demographic history of C. argyrosperma during its domestication}

We eliminated the SNPs that deviated from Hardy-Weinberg equilibrium (exact test with $p<0.01$ ) and pruned nearby SNPs under linkage disequilibrium (LD with an $r^{2}>0.25$ in $100 \mathrm{kbp}$ sliding windows) from the $13 \mathrm{k}$ dataset to retrieve a set of 2861 independent SNPs that could be used for demographic analyses.

We found similar genetic variation in sororia and argyrosperma, regardless of the reference genome used (average nucleotide diversity $\pi$ range $0.095-0.098$ for both taxa, see Table S4). At a population scale, the wild population in Jalisco had the highest genetic diversity within sororia, while the highest diversity in argyrosperma was found in the Pacific Coast of Mexico (Table S5). The domesticated and wild populations of C. argyrosperma displayed low genetic differentiation $\left(F_{\mathrm{ST}}=0.0646 ; 95 \%\right.$ confidence interval from 0.0565 to 0.0751 ), while feral populations were more closely related to argyrosperma $\left(F_{\mathrm{ST}}=0.0479\right)$ than with sororia $\left(F_{\mathrm{ST}}=0.1006\right)$.

We used SNPhylo ${ }^{21}$ and ADMIXTURE ${ }^{22}$ to evaluate the genealogical relationships and genetic structure among the wild and domesticated populations of C. argyrosperma. We confirmed the genetic differentiation between sororia and argyrosperma, as detected by the $F_{\mathrm{ST}}$ analyses. Our Maximum-Likelihood (ML) tree groups all the argyrosperma populations in a single monophyletic clade (Fig. 3a). We found additional genetic differentiation between the sororia populations in Southern Mexico (populations 1-3) and the sororia populations in Jalisco (population 4), in both the ADMIXTURE assignations (Fig. 3b) and their positions in the ML tree (Fig. 3a). The sororia populations of Jalisco are genetically closer to argyrosperma, as shown by their paraphyletic position in the ML tree (Fig. 3a). Consistent with a domestication in the lowlands of Western Mexico, the argyrosperma populations of Guerrero and Jalisco represent the basal branches of the argyrosperma clade (Fig. 3a), all showing instances of genetic similarity to the sororia populations in Jalisco in the four genetic groups $(K)$ of ADMIXTURE (Fig. 3b). The argyrosperma populations in Western Mexico (populations 5-17) are genetically differentiated from the Eastern populations (populations 18-26), with a possible recent anthropogenic dispersion event of Eastern populations into Onavas, Sonora (population 19; Fig. 3b, c). These four 
genetic groups are also retrieved in a principal component analysis (PCA; Fig. S2). The ADMIXTURE results (Fig. 3b) uncovered admixture events between some sororia and argyrosperma populations. This pattern is evident in the populations of Oaxaca and Sinaloa (populations 14 and 18; Fig. 3c). The feral populations are consistently grouped alongside their sympatric domesticated populations within argyrosperma (Fig. 3a, b), indicating that these populations diverged recently from nearby domesticated populations. These demographic patterns are robust to different SNP filters, such as different thresholds for missing data, or filtering for significant deviations from Hardy-Weinberg equilibrium (Fig. S3).

We used Fastsimcoal $2^{23}$ to explicitly test whether argyrosperma was domesticated from a sororia population in Southern Mexico or from a sororia population in Jalisco. Given that gene flow has been previously observed between argyrosperma and sororia ${ }^{12}$, we compared three different gene flow models (continuous gene flow, secondary contact, or no gene flow) for each scenario (Fig. 4a). A comparison between models using the Akaike Information Criterion indicates that the Jalisco domestication model with secondary contact (i.e., extant gene flow after initial genetic isolation between subspecies) is the most likely of the domestication scenarios assayed. We were able to discard the other unrealistic domestication scenarios (i.e., domestication in southern Mexico and lack of gene flow between subspecies), further supporting a domestication event in Jalisco.

\section{Selection scans in C. argyrosperma}

In order to perform the tests to detect selective signals associated with the domestication of $C$. argyrosperma, we removed from the $13 \mathrm{k}$ dataset the $C$. moschata individuals as well as the feral individuals of $C$ argyrosperma. We used the 1\% MAF threshold for this subset, obtaining a 10,617 SNP dataset suitable to detect selective signals, with a marker density of 44 SNPs per Mb. LD was limited within the dataset, with a mean pairwise $r^{2}$ of 0.1 , and with argyrosperma showing a faster LD decay than sororia (Fig. S4). We performed three outlier tests as implemented in BayeScEnv ${ }^{24}$, PCAdapt ${ }^{25}$, and LFMM $2^{26,27}$ to detect selective signals between the domesticated and the wild populations of $C$. argyrosperma (Fig. 5). BayeScEnv is an $F_{\mathrm{ST}}$-based method that tests correlation with other variables, in our case the wild or domesticated nature of each population (coded as 0 and 1 , respectively). PCAdapt does not require an a priori grouping of individuals into wild/domesticated, as we used the two principal components of a PCA to control for the underlying genetic structure between subspecies (Fig. S2). LFMM 2 identifies the number of latent factors in the populations through least square estimates to find correlations between genetic variants and the domesticated or wild phenotypes in the

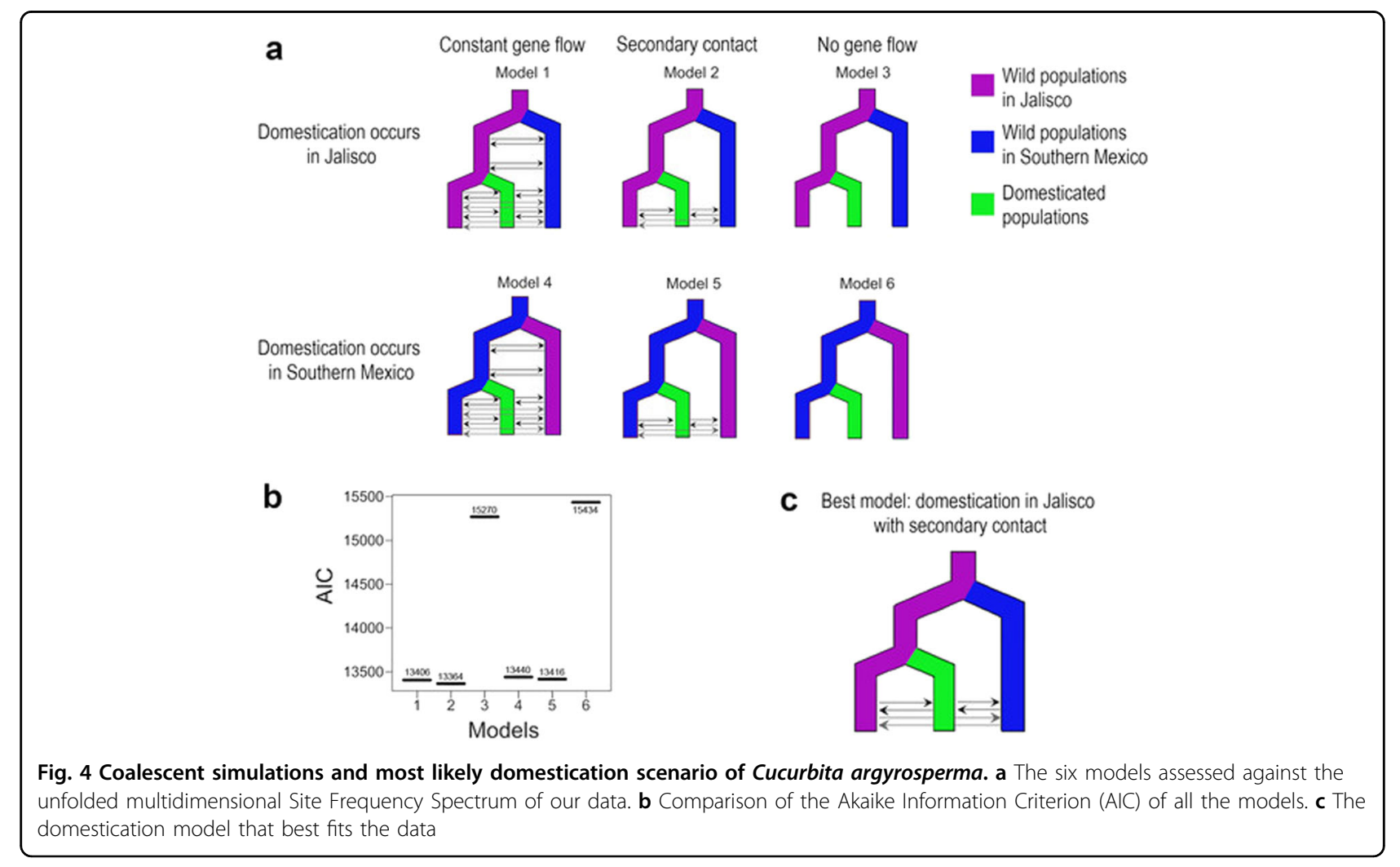




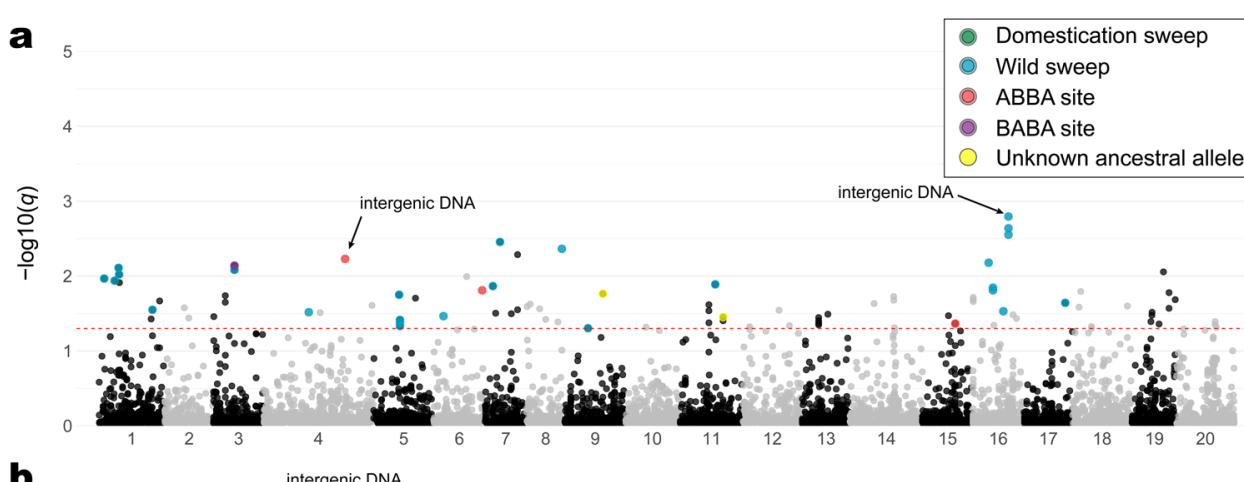

b . - intergenic DNA

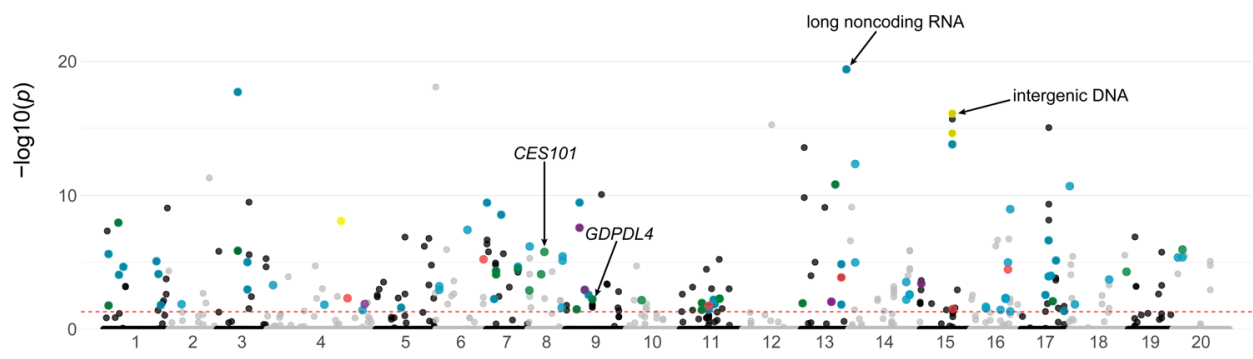

C 25

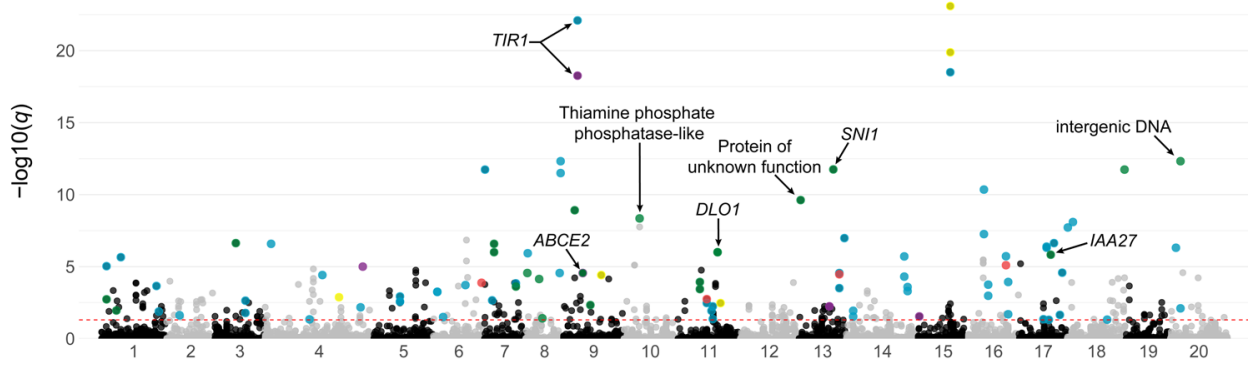

Chromosomes

Fig. 5 Putative footprints of selection associated with the domestication of Cucurbita argyrosperma. Manhattan plots representing the a BayeScEnv, $\mathbf{b}$ PCAdapt, and $\mathbf{c}$ LFMM 2 tests in each chromosome of the genome. The red dotted line indicates the cutoff value ( $q$-value or Bonferroni-corrected $p$-value $<0.05$ ) to determine a candidate locus. Only 110 loci were retrieved as candidate SNPs by more than one test, which are highlighted depending on whether the putative signal of positive selection corresponds to argyrosperma (green), sororia (blue), an ABBA site (red) a BABA site (purple) or an unknown selective direction (yellow). The arrows indicate the position of some of the discovered candidate genes

dataset ${ }^{26}$. In order to reduce the number of false positives, we only analyzed the SNPs that were detected as outliers by at least two of the tests.

We discovered 110 SNPs that converged as candidates in at least two tests (Fig. 5). We used C. moschata and C. okeechobeensis subsp. martinezii as outgroups to determine the direction of the putative selective pressures for each candidate SNP, as well as determining possible introgression or incomplete lineage sorting between sororia, argyrosperma and C. moschata. We found that 22 of the candidate SNPs corresponded to selective signals in argyrosperma, while 70 candidate SNPs corresponded to selective signals in sororia (Fig. 5). We could not determine the direction of selection for 5 candidate SNPs, and 13 showed signals of introgression or incomplete lineage sorting.
We identified several instances of either genetic introgression or incomplete lineage sorting between C. moschata and both argyrosperma (ABBA sites) or sororia (BABA sites) (Fig. S5). We performed a genomewide $D$-statistic analysis and found significantly more instances of shared derived variants between argyrosperma and $C$. moschata than between sororia and C. moschata (block-jackknife $p$-value $=0.0014$ ), obtaining an overall admixture fraction $f_{\mathrm{G}}$ of 0.01 (Table S6). From the 13 candidate SNPs with signals of introgression, 6 correspond to $\mathrm{ABBA}$ sites and 7 correspond to BABA sites (Fig. 5).

We found 45 protein-coding genes and one longnoncoding RNA including candidate SNPs within their structure (i.e., introns, exons, UTRs), which were assigned according to the observed direction of the putative 
selective signals (Table S7). Among the genes under putative selection in sororia were four serine/threonineprotein kinases, including PBL10 and PBL23 (Table S7). Among the genes under putative selection in argyrosperma, we found glycerophosphodiester phosphodiesterase GDPDL4, auxin-responsive protein $I A A 27, \mathrm{ABC}$ transporter E family member 2 (ABCE2), DMR6-like oxygenase 1 (DLO1), and serine/threonine-protein kinase CES101 (Table S7). We also found several genes under putative selection overlapping $\mathrm{ABBA}$ and $\mathrm{BABA}$ sites (Table S7). We found a transport inhibitor response 1 (TIR1) homolog under putative selection in both sororia and as a BABA site. Curiously, the gene $M K P 1$ was found under selection in both argyrosperma and in sororia.

\section{Discussion}

The genome assembly of sororia represents the first high-quality sequenced genome of a wild Cucurbita, which allowed us to detect structural and functional differences with the argyrosperma genome. The genome assembly of argyrosperma was smaller than the sororia assembly, which is possibly caused by the loss of structural variants during its domestication, as has been reported in pan-genome studies ${ }^{28}$. Many of these unaligned regions contain entire genes in sororia, making this wild taxon a reservoir of potentially adaptive presence/ absence variants. However, the extant genetic diversity of argyrosperma is similar to that of sororia, which suggests that the effects of the domestication bottleneck were alleviated by the current gene flow between both subspecies as suggested by our coalescent simulations and by the results of the previous studies ${ }^{12}$. The fast LD decay in argyrosperma further supports the limited effect of the domestication bottleneck. This gene flow may be related to the sympatric distribution of the wild and domesticated populations of $C$. argyrosperma throughout the Pacific Coast of Mexico ${ }^{11}$, where their coevolved pollinator bees Peponapsis spp. and Xenoglossa spp. are found ${ }^{29}$. Traditional agricultural practices are another fundamental force that maintains the diversity of crop species ${ }^{30}$. Since argyrosperma is a traditional crop cultivated for both selfsupply and local markets where it has a specialized gastronomic niche ${ }^{13}$, the genetic diversity in argyrosperma is also maintained by the conservation of local landrace varieties at local scales ${ }^{31,32}$.

Our demographic analyses suggest that the extant populations from Jalisco are the closest modern relatives of the initial population of sororia from which argyrosperma originated. The genetic relatedness between the sororia populations from Western Mexico and argyrosperma was also observed with mitochondrial markers ${ }^{6}$. The domestication of $C$. argyrosperma likely started around 8,700 years ago, as suggested by the earliest, albeit taxonomically ambiguous, archaeological record of argyrosperma ${ }^{10,33}$. Since crop domestication in Mesoamerica is linked to migration patterns and cultural development of early human populations in America ${ }^{7,34}$, we expected argyrosperma to share historical demography patterns with human history. Our data shows that the argyrosperma populations found in Guerrero and Jalisco are the most closely related to the sororia populations from Jalisco. This means that even if the closest wild relatives of argyrosperma are currently found in Jalisco, the domestication process may have occurred throughout the lowlands of Jalisco and the Balsas basin ${ }^{10}$. Ancient human migration events have been proposed to occur along the river basins in Southwestern Mexico, which may explain the genetic cohesiveness among the argyrosperma populations of that area that represent the first fully domesticated lineage of the species ${ }^{34}$. Previous studies based on 8,700 years old phytoliths found in Xihuatoxtla, Guerrero, suggest the co-occurrence of Zea mays and C. argyrosperma in the Balsas region within this time period $^{10,33}$. Overall, the patterns of genetic structure for C. argyrosperma are coherent with the archaeological evidence of early human migration throughout Mesoamerica $^{35,36}$ (Fig. 6).

We found several signals of putative selection between argyrosperma and sororia, even though tGBS sequencing has a limited capacity to detect selective signals across the genome $^{37}$. The SNP density for our selection tests was of 44 SNPs per $\mathrm{Mb}$, which is one order of magnitude denser than other studies using reduced-representation genome sequencing to detect selective signals ${ }^{37}$. This is a consequence of the relatively small genome size of $C$. argyrosperma $^{14}$. Nonetheless, the LD in C. argyrosperma decays at a shorter length than our SNP density, so several signals of selection might be missing from our scans, and some signals might not correspond to the actual gene under selection, but rather to a neighboring region in the genome $^{37}$. Therefore, our genome scans should be interpreted as a partial representation of the selective signals associated with the domestication process ${ }^{37}$.

Most of the putative selective signals were attributed to sororia, probably because wild taxa are subject to many natural selective pressures. Many of the SNPs that were retrieved as outliers were found on genes involved in biotic and abiotic plant defense responses. For example, PBL10 and PBL23 have been suggested to be involved in plant defense pathways due to their similarity to other serine/threonine-protein kinases ${ }^{38}$.

However, we also found some genes under putative selection in argyrosperma that could be attributed to defense mechanisms such as DLO1, which is involved in pathogenic defense responses ${ }^{39}$. We found GDPDL4 under putative selection, which is involved in trichome differentiation ${ }^{40}$, a morphological characteristic that differentiates argyrosperma from sororia (Fig. 1). We also 


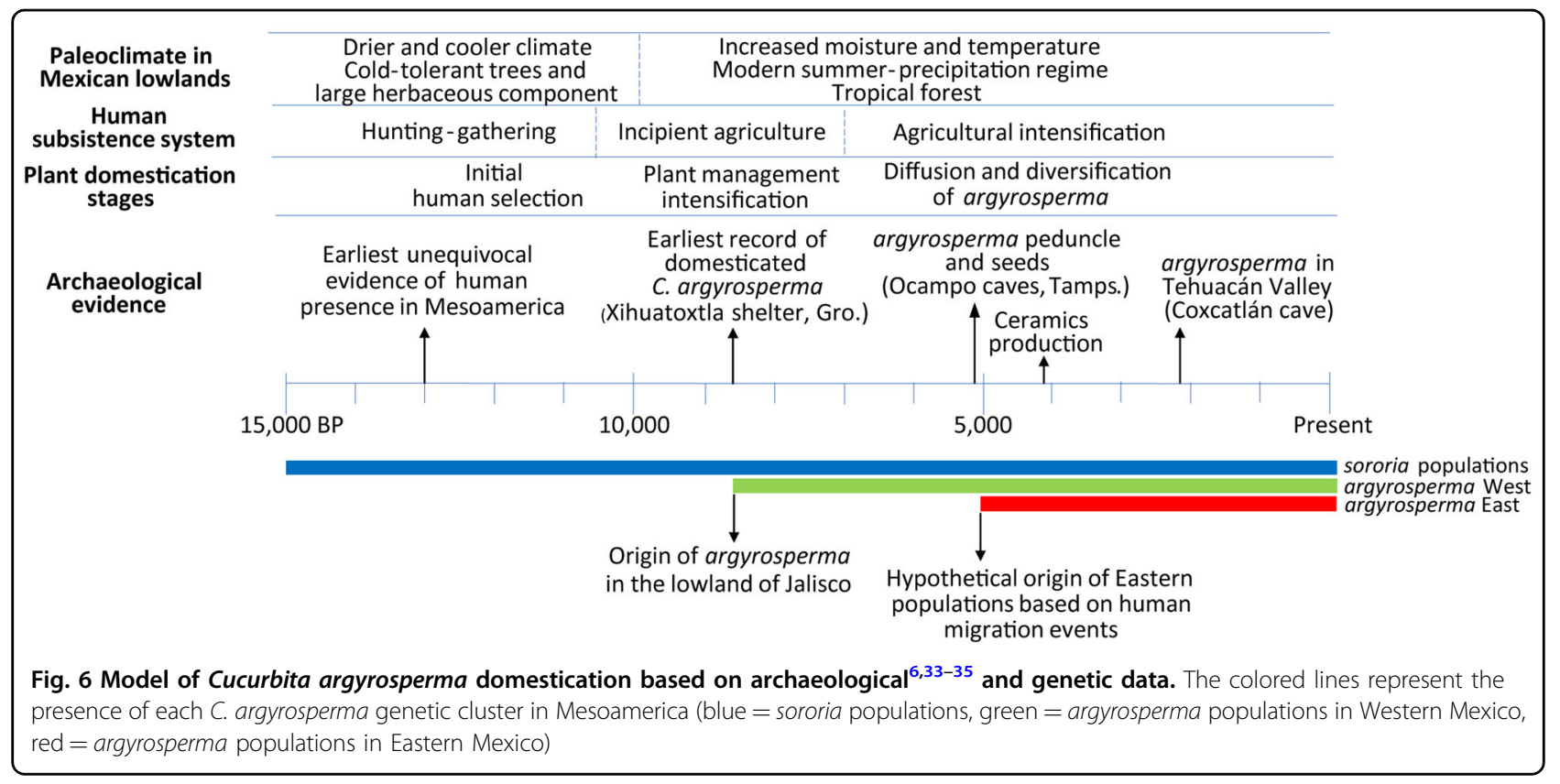

found two disrupted copies of TL1 and the absence of OLE6 within the argyrosperma genome, both of which cause allergic reactions in humans ${ }^{41,42}$. This is concordant with previous studies showing that selective pressures during domestication actively purge these defense mechanisms, as the products of these responses are usually unpleasant or harmful to humans when the plant is consumed ${ }^{43}$. This is particularly important for breeding programs, since wild Cucurbita such as sororia harbor loci associated with disease resistance that their domesticated counterparts have lost ${ }^{4}$. The selective pressures found in $M K P 1$ suggest a disruptive selection regime between sororia and argyrosperma. Since MKP1 modulates defense responses ${ }^{44}$, it is possible that both subspecies adapted to differential environmental pressures as domestication took place.

We found several candidate genes involved in the regulation of ABA. The alteration of growth hormones may play an important role in C. argyrosperma domestication. ABA is involved in a myriad of functions, such as the regulation of plant growth, plant development, seed dormancy, and response to biotic/abiotic stress ${ }^{45}$. In this sense, the lack of dormancy in seeds and gigantism are both common domestication changes that are present in domesticated cucurbits that may be caused by changes in the regulation of ABA and brassinosteroids ${ }^{8,46}$. We found SAUR32, PIF1, and LAF3 within the high-confidence SVs in argyrosperma, all of which act as inhibitors of seed germination under dark conditions ${ }^{47-49}$ and may explain the lack of seed dormancy in argyrosperma. Likewise, we found IAA27 under selection in argyrosperma, which is involved in plant growth and development ${ }^{50}$.
Selection over seed size has been particularly important in the domestication of $C$. argyrosperma ${ }^{9}$. We found $A B C E 2$ under selection in argyrosperma. Some $A B C$ transporters are involved in the transmembrane transport of ABA-GE, an ABA conjugate that is usually attributed to the plant response against water stress ${ }^{51}$. However, previous studies in Hordeum vulgare suggest that the transport of ABA-GE may play a role in seed development alongside de novo ABA synthesis within the developing seed $^{52}$, suggesting a role of $A B C$ transporters in the seed development of $C$. argyrosperma. Previous studies have also identified an association between variants in $\mathrm{ABC}$ transporter proteins and seed size in Cucurbita maxima ${ }^{53}$ and Linum usitatissimum ${ }^{54}$, further suggesting that ABA may be deregulated via selective pressures on $A B C$ transporters to enhance seed size in C. argyrosperma during its domestication. Variants in a serine/threonineprotein kinase, as the one we found in our selective scans, have also been associated with seed size in C. maxima ${ }^{53}$.

We found shared derived variants between $C$. moschata and $C$. argyrosperma under putative selection. This suggests that, given the close relationship between $C$. argyrosperma and $C$. moschata, both species may share domestication loci involved in common domestication traits. However, we also found TIR1 under selection in sororia and as a BABA site, which is an auxin receptor involved in ethylene signaling and antibacterial resistance in roots ${ }^{55}$. The introgression of wild alleles into domesticated Cucurbita has been previously reported as an effective method to improve the resistance of domesticated crops $^{56}$. Along this line, our results suggest that sororia is an important source of adaptive alleles for 
C. moschata. ABBA and BABA sites under selection may be shared with $C$. moschata either due to incomplete lineage sorting or by adaptive introgression with the wild and domesticated populations of $C$. argyrosperma. The incorporation of domesticated loci between $C$. argyrosperma and C. moschata through introgression may have been an effective way for Mesoamerican cultures to domesticate multiple Cucurbita taxa. This hypothesis is supported by the significant amount of ABBA sites shared between the genomes of $C$. argyrosperma and C. moschata. However, this hypothesis needs to be further addressed using population-level data of $C$. moschata with other wild and domesticated Cucurbita species.

\section{Materials and methods}

\section{Genome assembly and annotation of Cucurbita argyrosperma subsp. sororia}

We sequenced and assembled de novo the genome of a sororia individual collected in Puerto Escondido (Oaxaca, Mexico). Its DNA was extracted from leaf tissue and sequenced using PacBio Sequel at the University of Washington PacBio Sequencing Services and using Illumina HiSeq4000 at the Vincent J. Coates Genomics Sequencing Laboratory in UC Berkeley (NIH S10 Instrumentation Grants S10RR029668 and S10RR027303). We filtered the Illumina sequences using the qualityControl.py script (https://github.com/Czh3/NGSTools) to retain the reads with a PHRED quality $\geq 30$ in $85 \%$ of the sequence and an average PHRED quality $\geq 25$. The Illumina adapters were removed using SeqPrep (https://github.com/ jstjohn/SeqPrep) and the paired reads that showed overlap were merged. The chloroplast genome was assembled using NOVOplasty ${ }^{57}$ and the organellar reads were filtered using Hisat $2^{58}$ against the chloroplast genome of argyrosperma $^{14}$ and the mitochondrial genome of C. pepo ${ }^{59}$. We assembled the nuclear genome into small contigs using the Illumina reads and the Platanus assembler ${ }^{60}$. The Platanus contigs were assembled into larger contigs using the PacBio Sequel reads and DBG2OLC ${ }^{61}$. We performed two iterations of minimap2 and racon ${ }^{62}$ to obtain a consensus genome assembly by mapping the PacBio reads and the Platanus contigs against the DBG2OLC backbone. We performed three additional polishing steps using PILON ${ }^{63}$ by mapping the Illumina reads against the consensus genome assembly with BWA mem $^{64}$.

The genome annotation processes were performed using the GenSAS v6.0 online platform ${ }^{65}$. The transposable elements within the genome were predicted and masked using RepeatModeler (http://www.repeatmasker. org/RepeatModeler/). We downloaded five RNA-seq libraries of $C$. argyrosperma available on the Sequence Read Archive (accessions SRR7685400, SRR7685404SRR7685407) to use them as RNA-seq evidence for the gene prediction. We performed the same quality filters described above for the RNA-seq data and aligned the high-quality reads against the masked genome of $C$. argyrosperma subsp. sororia using STAR v2. $7^{66}$. We used filterBAM from the Augustus repository ${ }^{67}$ to filter lowquality alignments and used the remaining alignments as RNA-seq evidence to predict the gene models using BRAKER $2^{17}$. The gene predictions were functionally annotated using InterProScan ${ }^{68}$ and by aligning the gene models against the SwissProt database ${ }^{69}$ using BLASTp ${ }^{70}$ with an $e$-value $<1 \mathrm{e}^{-6}$. We performed a manual assessment of the predicted gene models to eliminate annotation artifacts.

\section{Anchoring the reference genomes into pseudomolecules}

We aimed to improve the genome assemblies of argyrosperma and sororia, which were assembled in 920 scaffolds ${ }^{14}$ and 817 contigs, respectively. Thus, we generated PacBio corrected reads from the published PacBio RSII reads of argyrosperma (NCBI SRA accession SRR7685401) and the PacBio Sequel reads of sororia (sequenced for this study at the University of Washington PacBio Sequencing Services) using $\mathrm{CANU}^{71}$. The macrosynteny of Cucurbita genomes is largely conserved between species ${ }^{72}$, so we performed a reference-guided scaffolding step using RaGOO $^{18}$ to anchor the genome assemblies of argyrosperma ${ }^{14}$ and sororia into pseudomolecules. We used the genome assembly of $C$. moschata ${ }^{16}$ as the reference genome for RaGOO ${ }^{18}$ and we used the PacBio corrected reads of each taxon to detect and correct misassemblies, using a gap size of $2600 \mathrm{bp}$ for chromosome padding (i.e., we filled the gaps between contigs with 2600 stretches of Ns, corresponding to the average gap length of the argyrosperma genome assembly). The chromosome numbers in both assemblies were assigned in correspondence to the genome assembly of C. moschata ${ }^{16}$.

\section{Structural variant analysis}

We evaluated the synteny between Cucurbita genomes using Synmap2 ${ }^{73}$. We predicted the SVs between argyrosperma and sororia using $\mathrm{SyRI}^{74}$ alongside nucmer ${ }^{75}$ with a minimum cluster length of $500 \mathrm{bp}$, an alignment extension length of $500 \mathrm{bp}$, a minimum match length of $100 \mathrm{bp}$ and a minimum alignment identity of $90 \%$. We also used Sniffles alongside NGMLR ${ }^{76}$ as an additional SV predictor, by aligning the argyrosperma PacBio reads against the genome assembly of sororia (only the SVs with a minimum support of 6 reads were retained). We only analyzed the SVs that were independently predicted by SyRI and Sniffles. Only the SVs that overlapped within a range of $\pm 100 \mathrm{bp}$ at the start and end positions of each prediction were retained as high-confidence SVs. Due to the limitations of read-mapping techniques to predict presence-absence variants, we only analyzed the unaligned regions predicted by SyRI for this type of variants. 
The gene content associated with each type of structural variant was considered either as the overlap between genes and variants (inversions and translocations) or as the genes contained entirely within the structural variants (CNVs and unaligned regions). We performed a Gene Ontology enrichment analysis using topGO and the weight01 algorithm ${ }^{77}$ to find enriched biological functions associated with each type of structural variant. We determined the significantly enriched biological functions by performing Fisher's exact test ( $p$-value $<0.05)$. We plotted the genome rearrangements and SVs between sororia and argyrosperma using $\mathrm{Smash}^{78}$ with a minimum block size of $100,000 \mathrm{bp}$, a threshold of 1.9 , and a context of 28.

\section{Data filtering and SNP genotyping}

We used previously collected seeds from 19 populations of argyrosperma landraces, four populations of sororia, and three feral populations ${ }^{11}$, covering most of the reported distribution of this species throughout Mexico $^{5}$ (Table S3). Each of the collected seeds came from a different maternal plant, in order to avoid signals of inbreeding. The seeds were germinated in a greenhouse and total DNA was extracted from fresh leaves using a DNeasy Plant MiniKit (Qiagen) of 192 individuals across the collected populations (Table S3), including five individuals of $C$. moschata to be used as outgroup. All 192 individuals were sequenced by Data2Bio LLC using the tunable Genotyping by Sequencing (tGBS) method $^{20}$ with an Ion Proton instrument and two restriction enzymes (Sau3AI/BfuCI and NspI). The wild and domesticated populations were randomly assigned to the plate wells before library preparation to avoid sequencing biases.

The raw reads of the tGBS sequencing were trimmed using $\mathrm{LUCY}^{79}$, removing bases with PHRED quality scores < 15 using overlapping sliding windows of $10 \mathrm{bp}$. Trimmed reads shorter than $30 \mathrm{bp}$ were discarded. The trimmed reads were mapped against the genome assembly of argyrosperma using segemeh $l^{80}$, since empirical studies suggest this read-mapping software outperforms others for Ion Torrent reads ${ }^{81}$. We only retained the reads that mapped uniquely to one site of the reference genome for subsequent analyses.

We used BCFtools ${ }^{82}$ for an initial variant calling step, retaining variants with at least 6 mapped reads per individual per site where the reads had a minimum PHRED quality score of 20 in the called base and a minimum mapping quality score of $20^{83}$. We used plink ${ }^{84}$ to perform additional filters, such as retaining only biallelic SNPs, retaining SNPs with no more than $50 \%$ of missing data, individuals with no more than $50 \%$ of missing data and sites with a minor allele frequency (MAF) of at least 1\% (13k dataset). After eliminating individuals with missing data, 109 individuals of argyrosperma, 44 individuals of sororia, 14 feral individuals and 5 individuals of $C$. moschata remained for the subsequent analyses. We repeated the SNP prediction using the reference genome of sororia to evaluate potential reference biases. We found a similar number of SNPs $(10,990)$ and comparable estimates of genetic diversity (see Table S4), suggesting that reference bias does not have a meaningful impact on our results. Thus, we employed the domesticated genome as the reference for the rest of the population analyses. We repeated our analyses using a separate filtering step of missing data for the domesticated and the wild populations, retrieving 84.18\% of the SNPs from the 13k dataset and obtaining the same results (Fig. S3).

In order to obtain an adequate SNP dataset to infer the demographic history of $C$. argyrosperma, we performed additional filters to the $13 \mathrm{k}$ dataset with plink ${ }^{84}$, including (i) the elimination of all the SNPs that diverged significantly $(p<0.01)$ from the Hardy-Weinberg equilibrium exact test ${ }^{85}$ to remove potential allelic dropouts, (ii) the elimination of adjacent SNPs with a squared correlation coefficient $\left(r^{2}\right)$ larger than 0.25 within $100 \mathrm{kbp}$ sliding windows with a step size of $100 \mathrm{bp}$. We repeated the demographic analyses without filtering the SNPs with significant deviations from Hardy-Weinberg equilibrium, obtaining the same results (Fig. S3).

We also generated a SNP dataset to detect selective signals associated with the domestication of $C$. argyrosperma by eliminating all the feral individuals of $C$. argyrosperma, which could not be assigned to either a wild or a domesticated population, as well as the five individuals of $C$. moschata. We also eliminated the SNP sites with more than $50 \%$ missing data and performed a MAF filter of $1 \%$ after reducing the number of individuals in the 13k dataset. The SNP density was calculated with VCFtools ${ }^{86}$ and the LD decay was calculated using plink ${ }^{84}$ with a minimum $r^{2}$ threshold of 0.001 .

We also sequenced the genome of a C. moschata individual from Chiapas (Mexico) and the genome of a $C$. okeechobeensis subsp. martinezii individual from Coatepec (Veracruz, Mexico) using the Illumina HiSeq4000 platform in UC Berkeley, to evaluate possible introgression and incomplete lineage sorting with $C$. argyrosperma. We downloaded the genome sequences of argyrosperma ${ }^{14}$ from the Sequence Read Archive (accessions SRR7685402 and SRR7685403). The Illumina whole-genome sequences were filtered using the same quality parameters as the ones used in the genome assembly of sororia (see above) and were aligned against the genome assembly of argyrosperma using BWA mem ${ }^{64}$. We only retained the reads that mapped uniquely to one site of the reference genome and retained only the biallelic sites with a sequencing depth $>=10$ reads per genome. 


\section{Population structure}

We used diveRsity ${ }^{87}$ to calculate the pairwise $F_{\mathrm{ST}}$ statistics, using 100 bootstraps to calculate the $95 \%$ confidence intervals. We estimated the genetic variation in the wild, domesticated and feral populations with STACKS $^{88}$. Using ADMIXTURE ${ }^{22}$, we evaluated the genetic structure among the sororia and argyrosperma populations, evaluating their individual assignment into one $(\mathrm{CV}$ error $=0.26205)$, two $(\mathrm{CV}$ error $=0.25587)$, three $(\mathrm{CV}$ error $=0.25806)$ and four $(\mathrm{CV}$ error $=0.26658)$ genetic groups $(K)$. We reconstructed a maximumlikelihood tree with SNPhylo ${ }^{21}$, based on substitutions per site between all the individuals with 100 bootstraps to assess the reliability of the tree topology. We used plink ${ }^{84}$ to perform a principal component analysis (PCA) using 10 principal components.

\section{Coalescent simulations}

We used Fastsimcoal $2^{23}$ to determine the parameters that maximize the composite likelihood of each model given the unfolded multidimensional SFS. The unfolded multidimensional SFS was obtained with $\mathrm{DADI}^{89}$, using 17 sororia individuals of Jalisco, 27 sororia individuals of Southern Mexico, 109 argyrosperma individuals and 5 C. moschata individuals as an outgroup to unfold the SFS. We ran 100,000 simulations with 20 replicates for each model (two divergence scenarios and three gene flow scenarios) using the following settings: a parameter estimation by Maximum Likelihood with a stopping criterion of 0.001 difference between runs, a minimum SFS count of 1 , a maximum of 40 loops to estimate the SFS parameters and a maximum of 200,000 simulations to estimate the SFS parameters. We also selected log-uniform priors for parameter estimations, setting times of divergence between 1000 and 200,000 generations (domestication times are expected to fall within this interval, given that the presumed most ancient evidence of the human presence in America is 33,000 years old ${ }^{90}$; while the split between the wild populations is expected to coincide with the extinction of the megafauna in America, which acted as the natural dispersers of wild Cucurbita during the Pleistocene $\left.{ }^{3}\right)$, effective population sizes $\left(N_{\mathrm{e}}\right)$ between 100 and 60,000 individuals and migration rates (m) between 0.0001 and 0.5 . The constant gene flow scenarios calculated a migration rate matrix throughout the simulation, from the present back to the common ancestral population of all lineages, with independent migration rates for each possible direction of gene flow. The secondary contact scenarios simulated a migration matrix only at the start of the simulation, before the coalescence event between the wild and domesticated lineages (see Data S1-S6 for detailed model parameters). We also constrained the times of divergence in all scenarios by forcing the domesticated taxa to diverge after the wild one. Each generation in the model corresponds to one calendar year, as this species displays an annual life cycle $^{13}$. The 20 replicates of each model converged to similar likelihoods, indicating that the simulations performed well. After corroborating that all replicates converged to similar likelihoods, we combined all replicates and retained all outputs that were above $95 \%$ of the likelihood distribution. We found that the Jalisco model of divergence with secondary contact had the lowest Akaike Information Criterion values for all the tested models.

\section{Tests to detect selective signals and introgression}

We used BayeScEnv ${ }^{24}$ to detect putative regions under selection that were differentiated between sororia and argyrosperma. For the "environmental" values used by BayeScEnv, we assigned each population as either wild (0) or domesticated (1). We ran two independent MCMC analyses with 20 initial pilot runs with a length of 10,000 generations and a main run with an initial burn-in of 100,000 generations and a subsequent sampling step for 100,000 generations sampling every 20 generations. We confirmed the convergence between both chains using the Gelman and Rubin statistic ${ }^{91}$. The SNPs with $q$-values $<$ 0.05 were regarded as candidate loci under selection.

The Mahalanobis distances implemented in PCAdapt ${ }^{25}$ were used to detect candidate SNPs after controlling for the first two principal components in our dataset, which correspond to the subspecies and geographical differentiation observed among the populations (see Fig. S2). We performed Bonferroni corrections to adjust the $p$-values obtained from PCAdapt and the SNPs with Bonferronicorrected $p$-values $<0.05$ were regarded as candidate loci under selection.

We used LFMM $2^{26}$ to identify candidate SNPs differentiating the wild and domesticated phenotypes of $C$. argyrosperma. We tested $K$ number of latent factors from 1 to 10 using $\mathrm{sNMF}^{27}$ and determined an optimal $K=6$. We used 6 latent factors and a ridge penalty to identify significant associations between the response (wild or domesticated phenotypes) and the genetic variants. Finally, we performed FDR corrections to obtain $q$-values, with $q$-values $<0.05$ being regarded as candidate loci under selection.

We performed an ABBA-BABA test using Dsuit ${ }^{92}$ against the $11,498,421$ whole-genome variants to evaluate signals of introgression or incomplete lineage sorting between argyrosperma, sororia and $C$. moschata, while using C. okeechobeensis subsp. martinezii as an outgroup. We calculated a global $D$-statistic by performing a SNPby-SNP analysis to determine the amount of ABBA and BABA sites throughout the entire genome. We also calculated local $D$-statistics within 500 SNP windows with a step size of 250 SNPs. We used the five tGBS data of C. moschata, as well as the whole-genome sequences 
of C. moschata and C. okeechobeensis subsp. martinezii, to define the ancestral state of each candidate locus from the selection scans and determine the direction of the selective signals or whether they corresponded to ABBA or BABA sites. We used snpEff ${ }^{33}$ to associate the candidate loci found in at least two tests with the genome annotation of argyrosperma ${ }^{14}$.

\section{Acknowledgements}

This work was funded by Comisión Nacional para el Conocimiento y Uso de la Biodiversidad (CONABIO) KE004 "Diversidad genética de las especies de Cucurbita en México e hibridación entre plantas genéticamente modificadas y especies silvestres de Cucurbita" and CONABIO PE001 "Diversidad genética de las especies de Cucurbita en México. Fase II. Genómica evolutiva y de poblaciones, recursos genéticos y domesticación" (both awarded to R.L-S. and L.E.E.). J.B-R. and G.S-V. are doctoral students from Programa de Doctorado en Ciencias Biomédicas and Programa de Doctorado en Ciencias Biológicas, Universidad Nacional Autónoma de México, and received fellowships 583146 and 292164 from CONACYT. We acknowledge the Doctorado en Ciencias Biológicas and the Doctorado en Ciencias Biomédicas for the support provided during the development of this project. We thank Jodi Lynn Humann for her technical support while using the GenSAS web server. Special thanks to Laura Espinosa-Asuar and Silvia Barrientos for their technical support. We thank Rodrigo García Herrera, head of the Scientific Computing Department at LANCIS, Instituto de Ecología UNAM, for running the HTC infrastructure we used for the genome assembly and chromosome anchoring. The population genomic analyses were carried out using CONABIO's computing cluster, which was partially funded by SEMARNAT through the grant "Contribución de la Biodiversidad para el Cambio Climático" to CONABIO. We acknowledge the technical support of MSc Emanuel Villafán and the resources for highperformance computing that the Institute of Ecology (INECOL) made available for conducting the functional gene annotations.

\section{Author details}

'Departamento de Ecología Evolutiva, Instituto de Ecología, Universidad Nacional Autónoma de México, Circuito Exterior s/n Anexo al Jardín Botánico, 04510 Ciudad de México, México. ${ }^{2}$ Department of Ecology and Evolutionary Biology, University of California, Irvine, CA 92697, USA. ${ }^{3}$ Departamento de Conservación de la Biodiversidad, El Colegio de la Frontera Sur, Villahermosa, Carretera Villahermosa-Reforma km 15.5 Ranchería El Guineo 2a sección, 86280 Villahermosa, Tabasco, México. ${ }^{4}$ Génétique Quantitative et Evolution - Le Moulon, Université Paris-Saclay, Institut National de Recherche pour I'Agriculture, l'Alimentation et l'Environnement, Centre National de la Recherche Scientifique, AgroParisTech, Gif-sur-Yvette 91190, France. ${ }^{5}$ UBIPRO, Facultad de Estudios Superiores Iztacala, Universidad Nacional Autónoma de México, Av. de los Barrios \#1, Col. Los Reyes Iztacala, Tlalnepantla, Edo. de Mex 54090, México

\section{Author contributions}

L.E.E., M.I.T., G.S-V., and J.B-R. designed the research; G.S-V. and G.C-M. collected the biological material; G.S-V. extracted DNA for the tGBS data; G.C-M. and G.S-V. coordinated the sequencing procedures of the tGBS data; J.B-R. and X.A-D. extracted DNA for the reference genome and coordinated its sequencing procedures; E.A-P. coordinated the administrative and lab work; J-B-R., J.A.A-L. and Y.T.G-G. performed the bioinformatic analyses; G.S-V., J.B-R., J.A.A-L., M.I.T., and L.E.E. analyzed the results; J.B-R., G.S-V., and L.E.E. drafted the manuscript; R.L-S. and L.E.E. obtained the funding. All authors revised the final version of the manuscript.

\section{Data availability}

All the raw sequencing data and genome assemblies are available in the National Center of Biotechnology Information under the BioProject accession PRJNA485527 (genome accesions SDJN00000000 and JAGKQH010000000; SRA accesions available in Table S3). The genome assemblies are also available in Figshare (https://doi.org/10.6084/m9.figshare.14370584.v1) and at the Cucurbit Genomics Database ${ }^{94}$.
Conflict of interest

The authors declare no competing interest.

Supplementary information The online version contains supplementary material available at https://doi.org/10.1038/s41438-021-00544-9.

Received: 9 November 2020 Revised: 3 March 2021 Accepted: 14 March 2021

Published online: 01 May 2021

\section{References}

1. Meyer, R. S. \& Purugganan, M. D. Evolution of crop species: genetics of domestication and diversification. Nat. Rev. Genet. 14, 840-852 (2013).

2. Zeder, M. A. Core questions in domestication research. Proc. Natl Acad. Sci. USA 112, 3191-3198 (2015).

3. Kistler, L. et al. Gourds and squashes (Cucurbita spp.) adapted to megafaunal extinction and ecological anachronism through domestication. Proc. Natl Acad. Sci. USA 112, 15107-15112 (2015).

4. Paris, H. S. in Genetics and Genomics of Cucurbitaceae 111-154 (Springer International Publishing, 2016). https://doi.org/10.1007/7397_2016 3.

5. Castellanos-Morales, G. et al. Historical biogeography and phylogeny of Cucurbita: Insights from ancestral area reconstruction and niche evolution. Mol. Phylogenet Evol. 128, 38-54 (2018).

6. Sanjur, O. I., Piperno, D. R., Andres, T. C. \& Wessel-Beaver, L. Phylogenetic relationships among domesticated and wild species of Cucurbita (Cucurbitaceae) inferred from a mitochondrial gene: implications for crop plant evolution and areas of origin. Proc. Natl Acad. Sci. USA 99, 535-540 (2002).

7. Zizumbo-Villarreal, D., Flores-Silva, A. \& Marín, P. C.-G. The Archaic Diet in mesoamerica: incentive for milpa development and species domestication Economic Bot. 66, 328-343 (2012).

8. Chomicki, G., Schaefer, H. \& Renner, S. S. Origin and domestication of Cucurbitaceae crops: insights from phylogenies, genomics and archaeology. New Phytol. 226, 1240-1255 (2019).

9. Whitaker, T. W. \& Cutler, H. C. Cucurbits and cultures in the Americas. Economic Bot. 19, 344-349 (1965).

10. Piperno, D. R., Ranere, A. J., Holst, I., Iriarte, J. \& Dickau, R. Starch grain and phytolith evidence for early ninth millennium B.P. maize from the Central Balsas River Valley, Mexico. Proc. Natl Acad. Sci. USA 106, 5019-5024 (2009).

11. Sánchez-de la Vega, G. et al. Genetic resources in the Calabaza Pipiana Squash (Cucurbita argyrosperma) in Mexico: genetic diversity, genetic differentiation and distribution models. Front Plant Sci. 9, 400 (2018).

12. Montes-Hernandez, S. \& Equiarte, L. E. Genetic structure and indirect estimates of gene flow in three taxa of Cucurbita (Cucurbitaceae) in western Mexico. Am. J. Bot. 89, 1156-1163 (2002)

13. Lira, R. et al. in Ethnobotany of Mexico 389-401 (Springer New York, 2016). https://doi.org/10.1007/978-1-4614-6669-7_15.

14. Barrera-Redondo, J. et al. The genome of Cucurbita argyrosperma (Silver-Seed Gourd) reveals faster rates of protein-coding gene and long noncoding RNA turnover and neofunctionalization within Cucurbita. Mol. Plant 12, 506-520 (2019).

15. Simão, F. A., Waterhouse, R. M., loannidis, P., Kriventseva, E. V. \& Zdobnov, E. M. BUSCO: assessing genome assembly and annotation completeness with single-copy orthologs. Bioinformatics 31, 3210-3212 (2015).

16. Sun, $H$. et al. Karyotype stability and unbiased fractionation in the PaleoAllotetraploid Cucurbita genomes. Mol. Plant 10, 1293-1306 (2017).

17. Hoff, K. J., Lange, S., Lomsadze, A., Borodovsky, M. \& Stanke, M. BRAKER1: unsupervised RNA-Seq-based genome annotation with GeneMark-ET and AUGUSTUS. Bioinformatics 32, 767-769 (2016)

18. Alonge, M. et al. RaGOO: fast and accurate reference-guided scaffolding of draft genomes. Genome Biol. 20, 224 (2019).

19. Whitaker, T. W. \& Bemis, W. P. Origin and evolution of the cultivated Cucurbita. Bull. Torrey Bot. Club 102, 362-368 (1975).

20. Ott, A. et al. $\mathrm{tGBS}^{\circledR}$ genotyping-by-sequencing enables reliable genotyping of heterozygous loci. Nucleic Acids Res. 45, e178 (2017).

21. Lee, T. H., Guo, H., Wang, X., Kim, C. \& Paterson, A. H. SNPhylo: a pipeline to construct a phylogenetic tree from huge SNP data. BMC Genomics 15, 162 (2014).

22. Alexander, D. H., Novembre, J. \& Lange, K. Fast model-based estimation of ancestry in unrelated individuals. Genome Res. 19, 1655-1664 (2009). 
23. Excoffier, L. \& Foll, M. Fastsimcoal: a continuous-time coalescent simulator of genomic diversity under arbitrarily complex evolutionary scenarios. Bioinformatics 27, 1332-1334 (2011).

24. de Villemereuil, P. \& Gaggiotti, O. E. A new $F_{\text {ST }}$ based method to uncover local adaptation using environmental variables. Methods Ecol. Evol. 6, 1248-1258 (2015).

25. Luu, K., Bazin, E. \& Blum, M. G. PCAdapt: an R package to perform genome scans for selection based on principal component analysis. Mol. Ecol. Resour. 17, 67-77 (2017).

26. Caye, K., Jumentier, B., Lepeule, J. \& François, O. LFMM 2: fast and accurate inference of gene-environment associations in genome-wide studies. Mol. Biol. Evol. 36, 852-860 (2019).

27. Frichot, E., Mathieu, F., Trouillon, T., Bouchard, G. \& François, O. Fast and efficient estimation of individual ancestry coefficients. Genetics 196, 973-983 (2014).

28. Khan, A. W. et al. Super-Pangenome by integrating the wild side of a species for accelerated crop improvement. Trends Plant Sci. 25, 148-158 (2020).

29. Wilson, H. D. Gene flow in squash species. BioScience 40, 449-455 (1990).

30. Jarvis, D. I. et al. A global perspective of the richness and evenness of traditional crop-variety diversity maintained by farming communities. Proc. Natl Acad. Sci. USA 105, 5326-5331 (2008).

31. Montes-Hernández, S., Merrick, L. C. \& Eguiarte, L. E. Maintenance of squash (Cucurbita spp.) landrace diversity by farmers activities in Mexico. Genet. Resour. Crop Evol. 52, 697-707 (2005).

32. Barrera-Redondo, J. et al. Landrace diversity and local selection criteria of domesticated squashes and gourds (Cucurbita) in the central Andean mountain range of Peru: Tomayquichua, Huánuco. Bot. Sci. 98, 101-116 (2020).

33. Ranere, A. J., Piperno, D. R., Holst, I., Dickau, R. \& Iriarte, J. The cultural and chronological context of early Holocene maize and squash domestication in the Central Balsas River Valley, Mexico. Proc. Natl Acad. Sci. USA 106 5014-5018 (2009).

34. Zizumbo-Villarreal, D. \& Colunga-GarcíaMarín, P. Origin of agriculture and plant domestication in West Mesoamerica. Genet. Resour. Crop Evol. 57, 813-825 (2010).

35. Stinnesbeck, W. et al. The earliest settlers of Mesoamerica date back to the late Pleistocene. PLOS ONE 12, e0183345 (2017).

36. Piperno, D. R. The origins of plant cultivation and domestication in the new world tropics. Curr. Anthropol. 52, S453-S470 (2011).

37. Lowry, D. B. et al. Responsible RAD: striving for best practices in population genomic studies of adaptation. Mol. Ecol. Resour. 17, 366-369 (2017).

38. Zhang, J. et al. Receptor-like cytoplasmic kinases integrate signaling from multiple plant immune receptors and are targeted by a Pseudomonas syringae effector. Cell Host Microbe 7, 290-301 (2010).

39. Zeilmaker, T. et al. DOWNY MILDEW RESISTANT 6 and DMR6-LIKE OXYGENASE 1 are partially redundant but distinct suppressors of immunity in Arabidopsis. Plant J. 81, 210-222 (2015)

40. Hayashi, S. et al. The glycerophosphoryl diester phosphodiesterase-like proteins SHV3 and its homologs play important roles in cell wall organization. Plant Cell Physiol. 49, 1522-1535 (2008).

41. de Jesús-Pires, C. et al. Plant thaumatin-like proteins: function, evolution and biotechnological applications. Curr. Protein Pept. Sci. 21, 36-51 (2020).

42. Batanero, E., Ledesma, A., Villalba, M. \& Rodríguez, R. Purification, amino acid sequence and immunological characterization of Ole e 6, a cysteine-enriched allergen from olive tree pollen. FEBS Lett. 410, 293-296 (1997).

43. Moreira, X., Abdala-Roberts, L., Gols, R. \& Francisco, M. Plant domestication decreases both constitutive and induced chemical defenses by direct selection against defensive traits. Sci. Rep. 8, 12678 (2018).

44. Ulm, R. et al. Distinct regulation of salinity and genotoxic stress responses by Arabidopsis MAP kinase phosphatase 1. EMBO J. 21, 6483-6493 (2002).

45. Chen, $K$. et al. Abscisic acid dynamics, signaling, and functions in plants. J. Integr. Plant Biol. 62, 25-54 (2020).

46. Martínez, A. B. et al. Differences in seed dormancy associated with the domestication of Cucurbita maxima: elucidation of some mechanisms behind this response. Seed Sci. Res. 28, 1-7 (2017).

47. Park, J. E., Kim, Y. S., Yoon, H. K. \& Park, C. M. Functional characterization of a small auxin-up RNA gene in apical hook development in Arabidopsis. Plant Sci. 172, 150-157 (2007).

48. Yang, L., Jiang, Z., Jing, Y. \& Lin, R. PIF1 and RVE1 form a transcriptional feedback loop to control light-mediated seed germination in Arabidopsis. J. Integr. plant Biol. 62, 1372-1384 (2020).
49. Hare, P. D., Møller, S. G., Huang, L. F. \& Chua, N. H. LAF3, a novel factor required for normal phytochrome A signaling. Plant Physiol. 133, 1592-1604 (2003).

50. Liscum, E. \& Reed, J. W. Genetics of Aux/IAA and ARF action in plant growth and development. Plant Mol. Biol. 49, 387-400 (2002).

51. Burla, B. et al. Vacuolar transport of abscisic acid glucosyl ester is mediated by ATP-binding cassette and proton-antiport mechanisms in Arabidopsis. Plant Physiol. 163, 1446-1458 (2013).

52. Seiler, $C$. et al. ABA biosynthesis and degradation contributing to $A B A$ homeostasis during barley seed development under control and terminal drought-stress conditions. J. Exp. Bot. 62, 2615-2632 (2011).

53. Wang, $Y$. et al. Construction of a high-density genetic map and analysis of seed-related traits using specific length amplified fragment sequencing for Cucurbita maxima. Front Plant Sci. 10, 1782 (2019).

54. Guo, D. et al. Resequencing 200 flax cultivated accessions identifies candidate genes related to seed size and weight and reveals signatures of artificial selection. Front Plant Sci. 10, 1682 (2019).

55. Navarro, L. et al. A plant miRNA contributes to antibacterial resistance by repressing auxin signaling. Science 312, 436-439 (2006).

56. Holdsworth, W. L., LaPlant, K. E., Bell, D. C., Jahn, M. M. \& Mazourek, M. Cultivar-based introgression mapping reveals wild species-derived Pm0 , the major powdery mildew resistance locus in squash. PlOS ONE 11 e0167715 (2016)

57. Dierckxsens, N., Mardulyn, P. \& Smits, G. NOVOPlasty: de novo assembly of organelle genomes from whole genome data. Nucleic Acids Res. 45, e18 (2017).

58. Kim, D., Paggi, J. M., Park, C., Bennett, C. \& Salzberg, S. L. Graph-based genome alignment and genotyping with HISAT2 and HISAT-genotype. Nat. Biotechnol. 37, 907-915 (2019).

59. Alverson, A. J. et al. Insights into the evolution of mitochondrial genome size from complete sequences of Citrullus lanatus and Cucurbita pepo (Cucurbitaceae). Mol. Biol. Evol. 27, 1436-1448 (2010)

60. Kajitani, R. et al. Efficient de novo assembly of highly heterozygous genomes from whole-genome shotgun short reads. Genome Res. 24 1384-1395 (2014).

61. Ye, C., Hill, C. M., Wu, S., Ruan, J. \& Ma, Z. S. DBG2OLC: efficient assembly of large genomes using long erroneous reads of the third generation sequencing technologies. Sci. Rep. 6, 31900 (2016).

62. Li, H. Minimap2: pairwise alignment for nucleotide sequences. Bioinformatics 34, 3094-3100 (2018)

63. Walker, B. J. et al. Pilon: an integrated tool for comprehensive microbial variant detection and genome assembly improvement. PLOS ONE 9, e112963 (2014).

64. Li, H. \& Durbin, R. Fast and accurate long-read alignment with BurrowsWheeler transform. Bioinformatics 26, 589-595 (2010).

65. Humann, J. L., Lee, T., Ficklin, S. \& Main, D. Structural and functional annotation of eukaryotic genomes with GenSAS. Methods Mol. Biol. 1962, 29-51 (2019).

66. Dobin, A. et al. STAR: ultrafast universal RNA-seq aligner. Bioinformatics 29 , 15-21 (2013).

67. Stanke, M., Schöffmann, O., Morgenstern, B. \& Waack, S. Gene prediction in eukaryotes with a generalized hidden Markov model that uses hints from external sources. BMC Bioinform. 7, 62 (2006).

68. Jones, P. et al. InterProScan 5: genome-scale protein function classification. Bioinformatics 30, 1236-1240 (2014).

69. Schneider, M. et al. The UniProtKB/Swiss-Prot knowledgebase and its Plant Proteome Annotation Program. J. Proteom. 72, 567-573 (2009).

70. Camacho, C. et al. BLAST+: architecture and applications. BMC Bioinforma. 10, 421 (2009).

71. Koren, S. et al. Canu: scalable and accurate long-read assembly via adaptive kmer weighting and repeat separation. Genome Res. 27, 722-736 (2017).

72. Gong, L., Pachner, M., Kalai, K. \& Lelley, T. SSR-based genetic linkage map of Cucurbita moschata and its synteny with Cucurbita pepo. Genome 51, 878-887 (2008).

73. Haug-Baltzell, A., Stephens, S. A., Davey, S., Scheidegger, C. E. \& Lyons, E SynMap2 and SynMap3D: web-based whole-genome synteny browsers. Bioinformatics 33, 2197-2198 (2017).

74. Goel, M., Sun, H., Jiao, W. B. \& Schneeberger, K. SyRl: finding genomic rearrangements and local sequence differences from whole-genome assemblies. Genome Biol. 20, 277 (2019).

75. Kurtz, S. et al. Versatile and open software for comparing large genomes. Genome Biol. 5, R12 (2004).

76. Sedlazeck, F. J. et al. Accurate detection of complex structural variations using single-molecule sequencing. Nat. Methods 15, 461-468 (2018). 
77. Alexa, A., Rahnenführer, J. \& Lengauer, T. Improved scoring of functional groups from gene expression data by decorrelating $\mathrm{GO}$ graph structure. Bioinformatics 22, 1600-1607 (2006).

78. Pratas, D., Silva, R. M., Pinho, A. J. \& Ferreira, P. J. An alignment-free method to find and visualize rearrangements between pairs of DNA sequences. Sci. Rep. 5, 10203 (2015)

79. Li, S. \& Chou, H. H. LUCY2: an interactive DNA sequence quality trimming and vector removal tool. Bioinformatics 20, 2865-2866 (2004).

80. Hoffmann, S. et al. Fast mapping of short sequences with mismatches, insertions and deletions using index structures. PLoS Comput Biol. 5, e1000502 (2009).

81. Caboche, S., Audebert, C., Lemoine, Y. \& Hot, D. Comparison of mapping algorithms used in high-throughput sequencing: application to lon Torrent data. BMC Genomics 15, 264 (2014).

82. Li, $\mathrm{H}$. et al. The sequence alignment/map format and SAMtools. Bioinformatics 25, 2078-2079 (2009).

83. Li, H., Ruan, J. \& Durbin, R. Mapping short DNA sequencing reads and calling variants using mapping quality scores. Genome Res. 18, 1851-1858 (2008).

84. Purcell, S. et al. PLINK: a tool set for whole-genome association and population-based linkage analyses. Am. J. Hum. Genet. 81, 559-575 (2007).

85. Wigginton, J. E., Cutler, D. J. \& Abecasis, G. R. A note on exact tests of Hardy-Weinberg equilibrium. Am. J. Hum. Genet. 76, 887-893 (2005).
86. Danecek, P. et al. The variant call format and VCFtools. Bioinformatics 27 2156-2158 (2011)

87. Keenan, K., McGinnity, P., Cross, T. F., Crozier, W. W. \& Prodöhl, P. A. diveRsity: An $\mathrm{R}$ package for the estimation and exploration of population genetics parameters and their associated errors. Methods Ecol. Evolution 4, 782-788 (2013).

88. Catchen, J., Hohenlohe, P. A., Bassham, S., Amores, A. \& Cresko, W. A. Stacks: an analysis tool set for population genomics. Mol. Ecol. 22, 3124-3140 (2013).

89. Gutenkunst, R. N., Hernandez, R. D., Williamson, S. H. \& Bustamante, C. D. Inferring the joint demographic history of multiple populations from multidimensional SNP frequency data. PLoS Genet. 5, e1000695 (2009).

90. Ardelean, C. F. et al. Evidence of human occupation in Mexico around the Last Glacial Maximum. Nature 548, 87-92 (2020).

91. Gelman, A. \& Rubin, D. B. Inference from iterative simulation using multiple sequences. Stat. Sci. 7, 457-472 (1992).

92. Malinsky, M., Matschiner, M. \& Svardal, H. Dsuite-fast D-statistics and related admixture evidence from VCF files. Mol. Ecol. Resour. (2020).

93. Cingolani, P. et al. A program for annotating and predicting the effects of single nucleotide polymorphisms, SnpEff: SNPs in the genome of Drosophila melanogaster strain w1118; iso-2; iso-3. Fly (Austin) 6, 80-92 (2012).

94. Zheng, Y. et al. Cucurbit Genomics Database (CuGenDB): a central portal for comparative and functional genomics of cucurbit crops. Nucleic Acids Res. 47, D1128-D1136 (2019). 